Paraneoplastic limbic encephalitis with antibodies against intracytoplasmic antigens is not susceptible to immunotherapy.

\title{
HUMAN HERPESVIRUS 6 INFECTION AND FEBRILE SEIZURES
}

Frequency and clinical characteristics of primary human herpesvirus 6 (HHV-6) infection in hospitalized children with febrile seizures (FS) were investigated at Aghia Sophia Children's Hospital and Laiko General Hospital, Athens, Greece. Of 130 children with FS admitted during the study period, 65 returned for follow-up and were included; 55 had a first FS and 10 the second. The mean age was 21.35 months; 36 were male and 29 female. Using PCR in acute phase plasma and indirect immunofluorescent assay for antibody titers in acute and convalescent sera, primary HHV-6 infection was verified in $10(18 \%)$ with a first FS. Of 10 with primary HHV-6 infection, 6 had typical roseola, and 6 presented with respiratory or gastrointestinal symptoms. No child with a second episode of FS had primary HHV-6 infection. HHV-6 was type B in 8 children and type A in 2. HHV-6 DNA was not detected in 3 CSF samples examined. None of 85 control subjects had primary HHV-6 infection, but $49 \%$ had immunoglobulin $\mathrm{G}$ antibodies against the virus. Testing for HHV-6 infection is recommended as a routine laboratory test in young children admitted to the ED in Greece for first occurrence of a FS. (Laina I, Syriopoulou VP, Daikos GL et al. Febrile seizures and primary human herpesvirus 6 infection. Pediatr Neurol Jan 2010;42:28-31). (Respond: Dr Syriopoulou. E-mail: vsyriop@med.uoa.gr).

COMMENT. Primary HHV-6 infection is frequently associated with first febrile seizures (FS). HHV-6 infection is nearly universal in infancy or early childhood, and in the US it accounts for one third of all FS in children up to the age of 2 years. (Hall CB et al. New Engl J Med 1994;331:432-438). No increase or decreased incidence of recurrent FS occurs in children whose first FS is caused by HHV-6 vs other infections (Jee SH et al. Pediatr Inf Dis J 1998;17:43-48). The definitive role of HHV-6 infection in the etiology of FS is inconclusive, and multiple factors may be involved (Millichap JG, Millichap JJ. Pediatr Neurol 2006;35:165-172): 1) the height of the temperature (usually $>39 \mathrm{C}$ ); 2) encephalitis (mean frequency of virus detection in CSF is $14.5 \%$ of 138 samples in 9 studies of HHV-6 associated FS; range 0-100\%); and 3) viral neurotropism (HHV-6 encephalopathic factor). Further studies of CSF HHV-6 DNA are required. Evidence suggests that the generally accepted benign nature of FS may need modification, and the definition of FS changed to include a transitory encephalitic process in some more complex cases.

Magnetoencephalography showed equivalent current dipoles as evidence of focal interictal epileptic discharges in 8 of 15 children following a febrile seizure (Anninos $\mathrm{P}$ et al. J Child Neurol Jan 2010;25:61-66). The authors propose the MEG as a prognostic indicator of development of epilepsy in FS patients. 